\title{
Efficacy and Safety of Secukinumab in Chronic Plaque Psoriasis and Psoriatic Arthritis Therapy
}

\author{
Paolo Gisondi • Camilla Dalle Vedove $\cdot$ Giampiero Girolomoni
}

To view enhanced content go to www.dermtherapy-open.com

Received: November 29, 2013 / Published online: January 23, 2014

(c) The Author(s) 2014. This article is published with open access at Springerlink.com

\section{ABSTRACT}

Psoriasis is a chronic inflammatory skin disease affecting about $1-3 \%$ of the general population. Moderate-to-severe psoriasis is commonly associated with various comorbidities, including psoriatic arthritis (PsA) and cardiometabolic disorders such as obesity, hypertension, diabetes, and metabolic syndrome. There is increasing recognition that systemic inflammation accompanies severe skin disease. Abnormal innate and adaptive immune responses in the skin are involved in pathogenesis. The cytokine interleukin (IL)17A is produced by $\mathrm{T}$ helper 17 (Th17) cells, neutrophils, mast cells, and T cytotoxic 17 cells. IL-17 plays a key role in host defense against

Electronic supplementary material The online version of this article (doi:10.1007/s13555-014-0042-5) contains supplementary material, which is available to authorized users.

P. Gisondi $(\bowtie) \cdot$ C. Dalle Vedove · G. Girolomoni Section of Dermatology and Venereology,

Department of Medicine, University of Verona,

Verona, Italy

e-mail: paolo.gisondi@univr.it extracellular bacteria and fungi. IL-17A acts on keratinocytes to increase expression of chemokines involved in recruiting myeloid dendritic cells, Th17 cells, and neutrophils to the lesion site. IL-17A also induces the production of antimicrobial peptides and proinflammatory cytokines that, in turn, may amplify and sustain immune responses in the skin. Blocking IL-17A improved psoriasis-like pathology in experimental models, and reduction in IL-17 signaling is part of the mechanism of action of tumor necrosis factor- $\alpha$ blockers. Three agents neutralizing IL-17 (i.e., secukinumab and ixekizumab) or antagonizing its receptor (i.e., brodalumab) are currently being tested for efficacy and safety in the treatment of plaque psoriasis and PsA. Secukinumab is a fully human IgG1 monoclonal antibody that selectively binds and neutralizes IL-17A whose efficacy in the therapy of chronic plaque psoriasis has been demonstrated in different phase II clinical trial. No new safety signals have emerged so far.

Keywords: Brodalumab; Chronic plaque psoriasis; Dermatology; Ixekizumab; Psoriasis; Psoriatic arthritis; Secukinumab 


\section{THE RATIONALE FOR TARGETING IL-17 IN PSORIASIS}

Plaque psoriasis is a chronic inflammatory skin disease affecting $1-3 \%$ of the general population causing a significant impairment of quality of life, particularly if diffuse and recalcitrant to treatments [1]. Psoriasis is commonly associated with multiple comorbidities including psoriatic arthritis (PsA), inflammatory bowel diseases (Crohn's disease and ulcerative colitis), cardio-metabolic disorders (such as myocardial infarction hypertension, obesity, diabetes, dyslipidemia, fatty liver disease, and hyperuricemia) [2-5]. The association between psoriasis and cardiometabolic comorbidities could be explained considering unhealthy life habits (i.e., smoking, over-eating, and sedentary life), the systemic nature of psoriasis-related inflammation, as well as common genetic and pathogenic pathways $[5,6]$. The current understanding of the psoriasis pathogenesis assigns central importance to an interaction between acquired and innate immunity [7]. At the onset of the disease, plasmocytoid dendritic cells are activated in the dermis and produce tumor necrosis factor (TNF)- $\alpha$ and interleukin (IL)-23, which promote the development of $\mathrm{T}$ helper (Th)1 and Th17 lymphocytes which largely infiltrate psoriatic skin [8]. Th17 cells are a recently identified cluster of differentiation (CD4) + helper $\mathrm{T}$ cell subset which produce mainly IL-17A, IL-17F, IL-6, and, to a lesser extent, TNF- $\alpha$ and IL-22, which in turn are responsible for inflammatory changes and epidermal hyperplasia [9]. Notably, TNF- $\alpha$ also synergizes with IL-17A to induce many psoriasis-related genes in human epidermal keratinocytes [10]. IL-17A is produced not only by Th17 cells, but also by neutrophils, mast cells, and $\mathrm{T}$ cytotoxic cells.
IL-17A promotes inflammation by several actions including: overexpression of antimicrobial peptides such as lipocalin2, human $\beta$-defensin 2 and S100A proteins; secretion of several CXCR1- or CXCR2-bearing neutrophil chemoattractants such as CXCL1, CXCL3, CXCL5, CXCL6, and CXCL8, leading to neutrophil accumulation in psoriatic epidermis; increased production of CCL20, which is essential for the recruitment of both CCR6expressing dendritic cells and $\mathrm{T}$ cells in lesional skin; high expression of intercellular adhesion molecule-1 on endothelial cells, which is essential for T-cell extravasation; dominant role in inducing the expression of psoriasis signature genes that are synergistically or additively co-regulated together with TNF- $\alpha$, including IL-19, a potent inducer of epidermal hyperplasia, IL-6 and IL-23A (IL-23p19) [11-13]. Blocking IL-17A results in amelioration of psoriasis-like pathology in animal models [14]. Secukinumab (Novartis Pharma AG, Basel,

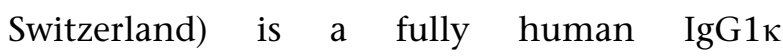
monoclonal antibody (mAb) that selectively binds and neutralizes IL-17A. Here, we report the efficacy and safety results of phase II clinical trials that investigated the efficacy and safety of secukinumab in the therapy of chronic plaque psoriasis and PsA.

The analysis in this article is based on previously conducted studies, and does not involve any new studies of human or animal subjects performed by any of the authors. Patient consent was obtained for the publication of the Fig. 1.

\section{SECUKINUMAB IN CHRONIC PLAQUE PSORIASIS}

Secukinumab demonstrated a favorable safety and tolerability profile with robust clinical activity, including improvements in psoriasis 

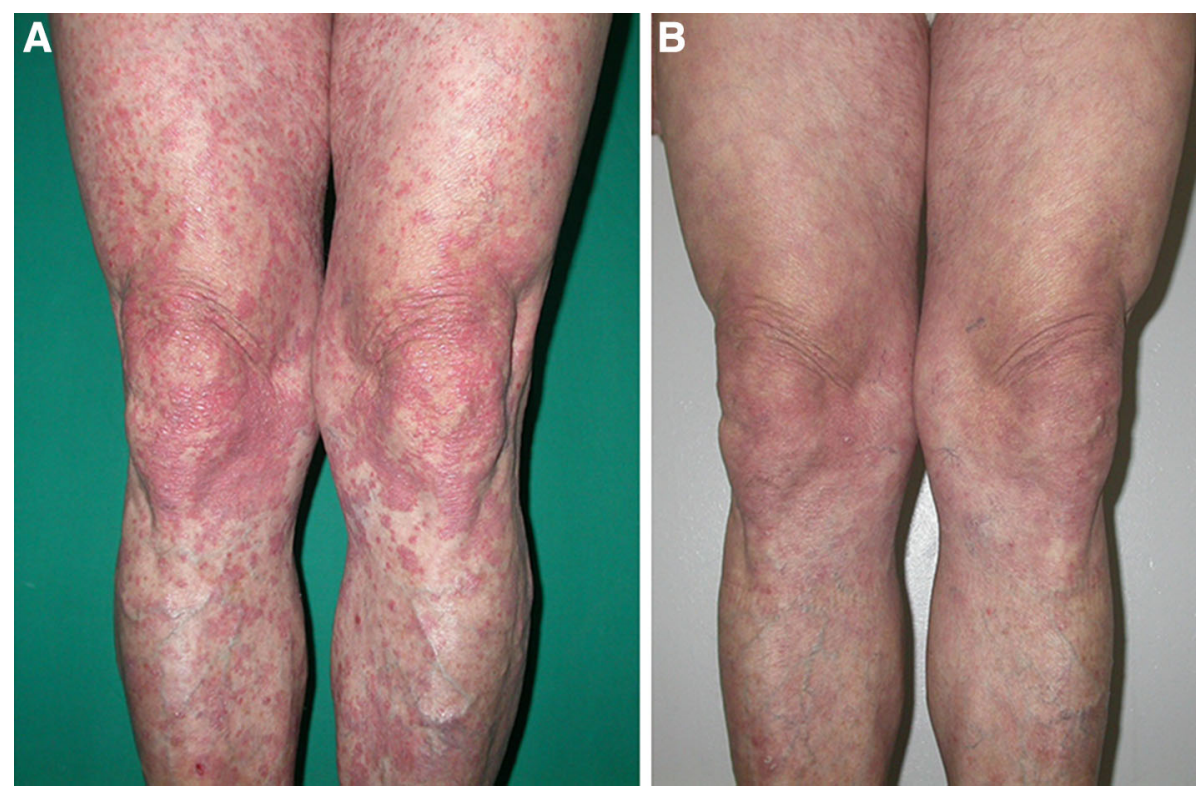

Fig. 1 Diffuse erythematous plaques of the lower limbs at baseline (a) and after 12 weeks of treatment with secukinumab $150 \mathrm{mg}$ subcutaneously week 0,4 , and 8 (b)

area and severity index (PASI) scores and investigator global assessment scores in patients with moderate-to-severe plaque psoriasis. A single infusion of secukinumab $(3 \mathrm{mg} / \mathrm{kg})$ resulted in rapid and sustained improvement of psoriasis in a double-blind, placebo-controlled, parallel-group, phase IIA proof-of-concept study on 36 patients [15]. At week 4, secukinumab was associated with an early significant reduction from baseline in mean PASI score compared to placebo (58\% vs. $4 \% ; \quad P=0.0001$ ) [15]. Accordingly, $83 \%$ of secukinumab patients versus $11 \%$ of placebo patients achieved significant decreases from baseline in investigator global assessment score $(P=0.0004)$ [15]. Benefits were maintained at week 12, with similar rates of adverse events (AEs), including infections, in both groups [15]. Consistent with the significant decrease in skin inflammation that was clinically evident, molecular profiling of psoriatic skin samples following treatment with secukinumab showed selective modulation of cytokines, including IL-12B， IL-17A， IL-17F， IL-21， IL-22， IL-26, interferon (IFN)- $\gamma$, and TNF- $\alpha$ [15]. These molecules are expressed by leukocyte and by tissue lineages, pointing to a diverse impact of IL-17A on multiple cellular lineages involved in psoriasis pathophysiology [15].

Secukinumab was further evaluated in phase IIB subcutaneous (SC) dose-ranging and intravenous (IV) regimen-finding studies. In particular, a randomized, double-blind, parallel-group, placebo-controlled, doseranging study evaluated four SC doses of secukinumab [16]. Patients $(n=125)$ were randomized 1:1:1:1:1 to receive doses of placebo $(n=22)$ or secukinumab $[1 \times 25 \mathrm{mg}$ $(n=29), \quad 3 \times 25 \mathrm{mg} \quad(n=26), \quad 3 \times 75 \mathrm{mg}$ $(n=21)$, or $3 \times 150 \mathrm{mg}(n=27)]$ at weeks 0,4 , and 8 . After the 12-week treatment, patients entered a follow-up period of 24 weeks. Secukinumab $150 \mathrm{mg}$ and $75 \mathrm{mg}$ (each administered at weeks 0,4 , and 8) were associated with significantly greater rates of PASI 75 response at week 12 compared with placebo (82\% and $57 \%$ vs. $9 \% ; P<0.001$ and $P=0.002$, respectively; Table 1 ). Results also 
indicated that doses of secukinumab higher than $75 \mathrm{mg}$ led to higher efficacy and higher doses of secukinumab may be required for some patients with greater body weight. Rates of AEs and serious AEs (SAEs) were comparable between secukinumab and placebo cohorts.

On the basis of the findings indicating the safety and efficacy of secukinumab $150 \mathrm{mg}$, a placebo-controlled phase IIB trial was conducted to assess 3 regimens of SC secukinumab $150 \mathrm{mg}$ in patients $(n=404)$ with moderate-to-severe plaque psoriasis, including single regimen (week $0 ; n=67$ ), early (weeks $0,1,2,4 ; n=133$ ), and monthly (weeks $0,4,8 ; n=138$ ) [17]. Early and monthly regimens resulted in significantly greater rates of PASI 75 response at week 12, the primary efficacy endpoint of the study, compared to placebo $(54.5 \%$ and $42 \%$ vs. $2 \% ; P<0.001$ for both comparisons; Table 1) [17]. The trial also investigated maintenance treatment strategies and secukinumab showed to offer patients and physicians the choice of either a fixed interval treatment regimen (every 4 weeks) or flexible dosing [17]. However, the maintenance period revealed a favorable efficacy profile of fixed interval period $(84.6 \%$ of patients) versus the on-demand regimen (67.2\%) in achieving PASI 75 at least once from week 20 to week 28 . In this study, an initial induction period (loading dosage) followed by the continuous treatment determined the optimal therapeutic regimen using SC $150 \mathrm{mg}$ secukinumab (Fig. 1). Secukinumab was associated with a safety profile comparable to placebo [17].

A similar phase IIB regimen-finding study was conducted to evaluate IV secukinumab induction regimens [18]. All secukinumab IV induction regimens were associated with significantly greater rates of PASI 75 and 90 responses at week 12 compared with placebo. Although there were six SAEs in the secukinumab treatment cohort, none were considered by the investigators to be drugrelated. Currently, secukinumab is being tested in several phase III clinical trials including a study evaluating efficacy of withdrawal-andretreatment, a study comparing secukinumab with etanercept, a study concerning moderateto-severe palmoplantar psoriasis and another trial on PsA [19].

\section{SECUKINUMAB IN PSORIATIC ARTHRITIS}

The efficacy and safety of secukinumab in patients with moderate-to-severe PsA was

Table 1 PASI 75 response rate at week 12 in secukinumab phase II clinical trials

\begin{tabular}{lllcc}
\hline Dose & $\begin{array}{l}\text { Weeks of subcutaneous } \\
\text { administration }\end{array}$ & $\begin{array}{l}\text { PASI 75 response } \\
\text { rate (\%) }\end{array}$ & $\begin{array}{l}\text { Sample } \\
\text { size }(\boldsymbol{N})\end{array}$ & References \\
\hline $1 \times 25 \mathrm{mg}$ & $0,4,8$ & 4 & 29 & {$[16]$} \\
$3 \times 25 \mathrm{mg}$ & $0,4,8$ & 18 & 26 & {$[16]$} \\
$3 \times 75 \mathrm{mg}$ & $0,4,8$ & 57 & 21 & {$[16]$} \\
$3 \times 150 \mathrm{mg}$ & $0,4,8$ & 82 & 27 & {$[16]$} \\
$150 \mathrm{mg}$ single & 0 & 10 & 67 & {$[17]$} \\
$150 \mathrm{mg}$ monthly & $0,4,8$ & 42 & 138 & {$[17]$} \\
$150 \mathrm{mg}$ early & $0,1,2,3,4$ & 54 & 133 & {$[17]$} \\
\hline$P$
\end{tabular}

$P A S I$ psoriasis area and severity index 
investigated in a 24-week, randomized, doubleblind, placebo-controlled, phase II proof-ofconcept trial [20]. Patients $(n=42)$ with active PsA were randomized (2:1) to receive two IV secukinumab doses $(10 \mathrm{mg} / \mathrm{kg} ; n=28)$ or placebo $(n=14) 3$ weeks apart. The primary objective of the study was the proportion of American College of Rheumatology 20 responses at week 6 for secukinumab versus placebo. At week 6, ACR20 responses were 39\% $(9 / 23)$ for secukinumab versus $23 \%$ (3/13) for placebo $(P=0.27)$. In contrast, no differences were registered for ACR 20 between secukinumab versus placebo at week 12 and week 24 [19]. At week 6, 'good' European League Against Rheumatism response was seen in $21.7 \%(5 / 23)$ secukinumab versus $9.1 \%$ $(1 / 11)$ in placebo group. Compared with placebo at week 6 , significant reductions were observed among secukinumab patients for $\mathrm{C}$ reactive protein $(P=0.039)$, erythrocyte sedimentation rate $(P=0.038)$, Health Assessment Questionnaire Disability Index $(P=0.002)$ and Short Form Health Survey (SF$36 ; P=0.030)$ scores. The overall AE frequency was similar between secukinumab and placebo. Six SAEs were reported in four secukinumab patients and one SAE in one placebo patient. Clinical responses, acute-phase reactant and quality of life improvements were greater with secukinumab versus placebo, although the primary endpoint was not achieved. Phase III clinical trials investigating secukinumab in PsA are ongoing.

\section{SAFETY OF SECUKINUMAB IN CHRONIC PLAQUE PSORIASIS AND PSA}

The crucial role of IL-17 in the innate immune response, poses IL-17 as the main driver of the host defense against extracellular bacterial (Staphylococcus aureus, Klebsiella pneumoniae, Streptococcus pneumoniae), parasitic (Toxoplasma gondii) and fungal (Candida spp.) infections [2123]. Importantly, both IL-17A and IL-17F are needed for muco-cutaneous immunity against Candida albicans, but their effects are largely redundant. Genetic deficiency in IL-17RA, in humans, is associated with recurrent or persistent muco-cutaneous infections caused by C. albicans and, to a lesser extent, S. aureus [24].

In the aforementioned studies, secukinumab was well tolerated, with an overall good safety profile; none of the patients developed antisecukinumab antibodies and there were no reports of injection-site reactions. Most of the AEs were mild or moderate in severity, particularly infections (Table 2) [16-18]. Vaccination against influenza is currently recommended to patients suffering from psoriasis [25]; previous studies have shown that vaccination against influenza virus and pneumococcus is safe with concomitant treatment with biologics like adalimumab and etanercept, and that it induces a satisfactory humoral response, whereas other agents, like rituximab, impaired vaccine effectiveness [26, 27]. Chioato et al. [28] have shown that blockade of IL-17A by secukinumab does not interfere with efficacy of influenza and meningococcal vaccinations, as assessed by the achievement of protective antibody levels.

\section{OTHER IL-17A INHIBITORS IN CHRONIC PLAQUE PSORIASIS}

Targeting of the IL-17A pathway for the treatment of psoriasis is being investigated with high interest now and other two agents that inhibit IL-17A are in phase III of clinical development, i.e., ixekizumab and brodalumab. 
Table 2 The most frequent adverse events (occurring in more than $5 \%$ of patients) reported in phase II clinical trial with different IL-17 inhibitors

\begin{tabular}{llll}
\hline Adverse event & \multicolumn{3}{l}{ Proportion of patients reporting the adverse event (\%) } \\
\cline { 2 - 4 } & Secukinumab [16, 17] & Ixekizumab [29] & Brodalumab [33] \\
\hline Worsening of psoriasis & $6.2-16.0$ & Not reported & Not reported \\
Nasopharyngitis & $12-22$ & $11-14$ & $3-16$ \\
Upper respiratory infection & $4.8-10.3$ & $4-10$ & $5-12$ \\
Headache & $3.4-9.1$ & $3-14$ & Not reported \\
Injection-site erythema & Not reported & $3-10$ & $3-10$ \\
\hline
\end{tabular}

IL-17 interleukin 17

Ixekizumab is a humanized IgG4 $\mathrm{mAb}$ neutralizing IL-17A. The safety and efficacy of ixekizumab was evaluated in a phase II, doubleblind, placebo-controlled trial with 142 patients affected by moderate-to-severe plaque-type psoriasis [29]. Patients were randomized into five groups receiving 150, 75, 25, $10 \mathrm{mg}$ ixekizumab or placebo, subcutaneously at 0,2 , $4,8,12$, and 16 weeks [29]. The achievement of $75 \%$ reduction of PASI 75 after 12 weeks occurred in $82.1 \%, 82.8 \%, 76.7 \%, 29.0 \%$, and $7.7 \%$ of patients, respectively. Regarding the safety profile, the occurrence of AEs, including nasopharyngitis, upper respiratory infection, injection-site reaction, and headache, was similar across all study groups, and no SAEs were reported (Table 2). Phase III studies are currently ongoing including a two head-to-head trials with etanercept and adalimumab in patients with PsA [30]. Ixekizumab is undergoing evaluation as a treatment for moderate-to-severe psoriasis in three phase III studies, UNCOVER-1 (Clinicaltrials.gov \#NCT01 474512), UNCOVER-2 (Clinicaltrials.gov \#NCT01597245), and UNCOVER-3 (Clinicaltrials.gov \#NCT01646177). These three studies have estimated completion dates between February 2018 and April 2019 [31].

Brodalumab is a human mAb blocking IL-17RA, the receptor subunit shared by
IL-17A, IL-17F, and IL-17A/F heterodimer ligands. The antagonism of IL-17 signaling by brodalumab was initially proven effective in inducing clinical, histologic, and genomic resolution of psoriasis after only 1 week of treatment in a phase I, proof-of-concept study enrolling 10 patients with psoriasis [32]. Further substantiating the efficacy is a more recent phase II, randomized, double-blind, placebo-controlled, dose-ranging study involving 198 patients randomly assigned to receive SC brodalumab at the dosage of $280 \mathrm{mg}$ monthly, or 70, 140, $210 \mathrm{mg}$ or placebo at weeks $0,1,2,4,6,8$, and 10 [33]. This 12 -week study assessed the efficacy of brodalumab in treating psoriasis with a mean percentage improvement of $45.0 \%, 85.9 \%, 86.3 \%, 76.0 \%$ and $16.0 \%$ of PASI score using $280,210,140$, $70 \mathrm{mg}$, or placebo, respectively $(P<0.001$ for all comparison vs. placebo) [33]. Common AEs occurred with greater frequency in the high-dose brodalumab group; among the SAEs, two cases of mild neutropenia (Grade III) were reported (Table 2) [33].

\section{CONCLUSION}

Psoriasis is facing very rapid advances in the understanding of its pathogenesis that drives a fast expanding availability of new therapeutic agents. Although anti-TNF- $\alpha$ drugs have 
markedly improved the therapeutic options of patients with autoimmune diseases, there is still a need for new treatment options for patients who lose response, fail to respond, or are intolerant to their current treatment [34]. More specific treatment options, with fewer side effects and a milder effect on the immune system, need to be identified. As more studies are performed, more clinical experience is gained, and more biomarkers of clinical response are identified, we will be able to determine which drugs are the most suitable for the long-term treatment of psoriasis. Pharmacogenetics studies may also help to tailor a more stratified therapeutic approach $[35,36]$. Experimental and clinical studies have identified IL-17 as a 'driver' cytokine of psoriasis and thus as an important therapeutic target. Based on good tolerability of IL-17 antagonists in phase II trials, it is expected that these agents are likely to become major future therapeutics for the treatment of psoriasis, although the exact place of these drugs needs further study. However, large-scale, phase III clinical trials are ongoing and they will provide informative data about the long-term efficacy and safety. Secukinumab is now in an advanced phase of development, but other anti-IL-17 agents have been designed and they, too, may expand the future therapeutic armamentarium. Still, the physiologic impact of long-term IL-17 antagonism will need to be demonstrated in larger and longer clinical trials and there might be important differences in antagonizing IL-17A versus the IL-17 receptor, which subtends broader cytokine effects. Also, there is a need for a better understanding of how IL-17 antagonism will impact PsA and other comorbidities that are associated with psoriasis and have a presumptive inflammatory etiology. Of these, the role of IL-17 in cardiovascular diseases holds special interest since there is increasing recognition that IL-17 is a major risk cytokine for atherosclerosis in model systems and that reduced expression or blockade of IL17 can reduce formation of atherosclerosis lesions [37].

\section{ACKNOWLEDGMENTS}

This work was supported by the Ministero della Salute, and the Ministero dell'Istruzione, Università e Ricerca Scientifica [Programmi di Ricerca Scientifica di Rilevante Interesse Nazionale (PRIN)], and by the Association for Dermatological Research (Italy). During the peer review process, the manufacturer of the agent under review was offered an opportunity to comment on the article. No comments were given and no changes were made as a result of this. All named authors meet the ICMJE criteria for authorship for this manuscript, take responsibility for the integrity of the work as a whole, and have given final approval for the version to be published.

Conflict of interest. P. Gisondi has received honoraria from lectures and/or board membership from Abbott, Janssen, Pfizer and Merck Sharp \& Dohme. C. Dalle Vedove declares no conflict of interest. G. Girolomoni has received honoraria from lectures, manuscript preparation, development of educational programs, and/or board membership from Abbott, Celgene, Actelion, Janssen, Merck-Serono, Pfizer, Merck Sharp \& Dohme, and Novartis.

Compliance with ethics guidelines. The analysis in this article is based on previously conducted studies, and does not involve any new studies of human or animal subjects performed by any of the authors. Patient 
consent was obtained for the publication of the Fig. 1.

Open Access. This article is distributed under the terms of the Creative Commons Attribution Noncommercial License which permits any noncommercial use, distribution, and reproduction in any medium, provided the original author(s) and the source are credited.

\section{REFERENCES}

1. Jacobson CC, Kumar S, Kimball AB. Latitude and psoriasis prevalence. J Am Acad Dermatol. 2011; 65:870-3.

2. Gisondi P, Tessari G, Conti A, et al. Prevalence of metabolic syndrome in patients with psoriasis: a hospital-based case-control study. Br J Dermatol. 2007;157:68-73.

3. Gisondi P, Targher G, Zoppini G, et al. Nonalcoholic fatty liver disease in patients with chronic plaque psoriasis. J Hepatol. 2009;51: 758-64.

4. Gisondi P, Targher G, Cagalli A, Girolomoni G. Hyperuricaemia in patients with chronic plaque psoriasis. J Am Acad Dermatol. 2014;70:127-30.

5. Lu Y, Chen H, Nikamo P, et al. Association of cardiovascular and metabolic disease genes with psoriasis. J Invest Dermatol. 2013;133:836-9.

6. Suárez-Fariñas M, Li K, Fuentes-Duculan J, Hayden $\mathrm{K}$, Brodmerkel C, Krueger JG. Expanding the psoriasis disease profile: interrogation of the skin and serum of patients with moderate-to-severe psoriasis. J Invest Dermatol. 2012;132:2552-64.

7. Nestle FO, Kaplan DH, Barker J. Psoriasis. N Engl J Med. 2009;361:496-509.

8. Albanesi C, Scarponi C, Pallotta S, et al. Chemerin expression marks early psoriatic skin lesions and correlates with plasmacytoid dendritic cell recruitment. J Exp Med. 2009;206:249-58.

9. Lowes MA, Kikuchi T, Fuentes-Duculan J, et al. Psoriasis vulgaris lesions contain discrete populations of Th1 and Th17 T cells. J Invest Dermatol. 2008;128:1207-11.

10. Ruddy MJ, Wong GC, Liu XK, et al. Functional cooperation between interleukin-17 and tumor necrosis factor-alpha is mediated by CCAAT/ enhancer-binding protein family members. J Biol Chem. 2004;27:2559-67.

11. Girolomoni G, Mrowietz U, Paul C. Psoriasis: rationale for targeting interleukin-17. $\mathrm{Br} \mathrm{J}$ Dermatol. 2012;167:717-24.

12. Gaffen SL. Structure and signalling in the IL-17 receptor family. Nat Rev Immunol. 2009;9: 556-67.

13. Chiricozzi A, Krueger JG. IL-17 targeted therapies for psoriasis. Expert Opin Investig Drugs. 2013;22: 993-1005.

14. Rizzo HL, Kagami S, Phillips KG, et al. IL-23mediated psoriasis-like epidermal hyperplasia is dependent on IL-17A. J Immunol. 2011;186: 1495-502.

15. Hueber W, Patel DD, Dryja $T$ et al. Effects of AIN457, a fully human antibody to interleukin$17 \mathrm{~A}$, on psoriasis, rheumatoid arthritis, and uveitis. Sci Transl Med. 2010;2:52-72.

16. Papp KA, Langley RG, Sigurgeirsson B, et al. Secukinumab efficacy and safety preliminary results from a phase II subcutaneous dose-ranging study in the treatment of moderate-to-severe plaque psoriasis. Br J Dermatol. 2013;168:412-21.

17. Rich P, Sigurgeirsson B, Thaci D, et al. Secukinumab induction and maintenance therapy in moderateto-severe plaque psoriasis: a randomized, doubleblind, placebo-controlled, phase II regimen-finding study. Br J Dermatol. 2013;168:402-11.

18. Papp KA, Matheson RT, Tu JH et al. Secukinumab, a novel fully human antibody to interleukin-17A, in the treatment of moderate-to-severe plaque psoriasis: efficacy and safety interim results from a phase II intravenous induction dose-ranging study. Presented at: 20th congress of the European Academy of Dermatology and Venereology; 20-24 October 2011, Lisbon, Portugal. Abstract 0630.

19. Clinicaltrials.gov. http://www.clinicaltrials.gov/ct2/ results?term=secukinumab+AND+psoriasis. Accessed December 13, 2013.

20. McInnes IB, Sieper J, Braun J, et al. Efficacy and safety of secukinumab, a fully human antiinterleukin-17A monoclonal antibody, in patients with moderate-to-severe psoriatic arthritis: a 24-week, randomised, double-blind, placebocontrolled, phase II proof-of-concept trial. Ann Rheum Dis. 2014;73:349-56.

21. Kelly MN, Kolls JK, Happel K, et al. Interleukin-17/ interleukin-17 receptor-mediated signaling is important for generation of an optimal 
polymorphonuclear response against Toxoplasma gondii infection. Infect Immun. 2005;73:617-21.

22. Huang $\mathrm{W}, \mathrm{Na}$ L, Fidel PL, Schwarzenberger P. Requirement of interleukin-17A for systemic antiCandida albicans host defense in mice. J Infect Dis. 2004;190:624-31.

23. Happel KI, Zheng M, Young E, et al. Cutting edge: roles of Toll-like receptor 4 and IL-23 in IL-17 expression in response to Klebsiella pneumoniae infection. J Immunol. 2003;170:4432-6.

24. Puel A, Cypowyj S, Bustamante J, et al. Chronic muco-cutaneous candidiasis in humans with inborn errors of interleukin-17 immunity. Science. 2011;332:65-8.

25. Lebwohl M, Bagel J, Gelfand JM, et al. From the Medical Board of the National Psoriasis Foundation: monitoring and vaccinations in patients treated with biologics for psoriasis. J Am Acad Dermatol. 2008;58:94-105.

26. Kaine JL, Kivitz AJ, Birbara C, et al. Immune responses following administration of influenza and pneumococcal vaccines to patients with rheumatoid arthritis receiving adalimumab. J Rheumatol. 2007;34:272-9.

27. Oren S, Mandelboim M, Braun-Moscovici Y, et al. Vaccination against influenza in patients with rheumatoid arthritis: the effect of rituximab on the humoral response. Ann Rheum Dis. 2008; 67:937-41.

28. Chioato A, Noseda E, Stevens M, et al. Treatment with the interleukin-17A-blocking antibody secukinumab does not interfere with the efficacy of influenza and meningococcal vaccinations in healthy subjects: results of an open label, parallelgroup, randomized single-center study. Clin Vaccine Immunol. 2012;19:1597-602.
29. Leonardi C, Matheson R, Zachariae C, et al. Antiinterleukin-17 monoclonal antibody ixekizumab in chronic plaque psoriasis. N Engl J Med. 2012;366: 1190-9.

30. Clinicatrials.gov. http://www.clinicaltrials.gov/ct2/ results?term=ixekizumab+AND+psoriasis. Accessed December 13, 2013.

31. Reichert JM. Which are the antibodies to watch in 2013? mAbs. 2013;5:1-4.

32. Russell CB, Kerkof K, Bigler J, et al. Blockade of the IL-17R with AMG827 leads to rapid reversal of gene expression and histopathologic abnormalities in human psoriatic skin. J Invest Dermatol. 2010;130: S46.

33. Papp KA, Leonardi C, Menter A, et al. Brodalumab, an anti-interleukin- 17-receptor antibody for psoriasis. N Engl J Med. 2012;366:1181-9.

34. Esposito M, Gisondi P, Cassano N, et al. Survival rate of anti-TNF alpha treatments for psoriasis in routine dermatological practice: a multicenter observational study. Br J Dermatol. 2013;. doi:10. 1111/bjd.12422.

35. Talamonti $\mathrm{M}$, Botti E, Galluzzo $\mathrm{M}$, et al. Pharmacogenetics of psoriasis: HLA-Cw6 but not LCE3B/3C deletion nor TNFAIP3 polymorphism predisposes to clinical response to interleukin 12/23 blocker ustekinumab. Br J Dermatol. 2013; 169:458-63.

36. Tejasvi T, Stuart PE, Chandran V, et al. TNFAIP3 gene polymorphisms are associated with response to TNF blockade in psoriasis. J Invest Dermatol. 2012;132(3 Pt 1):593-600.

37. Chen S, Crother TR, Arditi M. Emerging role of IL17 in atherosclerosis. J Innate Immun. 2010;2: 325-33. 